\title{
Fetal Findings Domain
}

National Cancer Institute

\section{Source}

National Cancer Institute. Fetal Findings Domain. NCI Thesaurus. Code C102637.

A subject domain utilized for the submission of information encompassing and representing data, vocabulary or records related to fetal findings. 